\title{
Innovationsföderalismus als Voraussetzung von Finanzverantwortung der Länder
}

von Christian Calliess

Die gegenwärtigen Bemühungen um eine „Föderalismusreform II“ gehen dahin, den Ländern mehr eingeständige finanzielle Verantwortung einzuräumen. Hierzu ist eine hinreichende Handlungsautonomie in Form von finanzwirksamen Gestaltungs- und Innovationsspielräumen erforderlich. Daher wird in diesem Beitrag für das Leitbild des „Innovationsföderalismus " plädiert, nach dem Spielräume geöffnet werden sollen, die wiederum zu einer Verbesserung der Effizienz des Gesamtsystems beitragen. Als konkrete Handlungsoptionen werden die Möglichkeiten einer Aufgabenoptimierung durch Koordinierung und „,benchmarking“ sowie einer erweiterten Standardsetzung, kombiniert mit dezentraler Flexibilisierung/Differenzierung, vorgestellt und auf ihre verfassungsrechtliche Machbarkeit hin untersucht. ${ }^{1}$

The ongoing discussion on a second stage in the reform of German federalism aims at enhanced fiscal responsibilities for the Länder. This implies the necessity of autonomous leeway in financial matters. Therefore, this contribution pleads for a new approach, labelled "innovative federalism", in form of a wider autonomy that ought to improve the efficiency of the whole system. As manageable options, the possibilities of an optimised performance through improved co-ordination and benchmarking as well as a combination of standardisation and decentral flexibilisation are being presented and examined regarding their constitutional feasibility.

\section{Einleitung}

Die sog. „,Föderalismusreform I“2 hatte Fragen der Finanzverfassung weitgehend ausgeklammert. Diese sollen nunmehr im Rahmen der sog. „Föderalismusreform II“ behandelt werden. In diesem Kontext geht es u. a. um die Novellierung des Länderfinanzausgleichs, die Einführung einer Schuldenobergrenze verbunden

1 Für die Unterstützung beim Verfassen dieses Beitrags dankt der Verfasser Herrn Dr. Stefan Korte. Der Beitrag geht zurück auf die Stellungnahme des Verfassers als Sachverständiger im Rahmen der öffentlichen Anhörung der Föderalismuskommission II am 8. November 2007. Sie entstand während seiner Tätigkeit am Institut für Völkerrecht und Europarecht der Universität Göttingen und profitierte vom dort gewährten Forschungsfreisemester.

2 Vgl. Häde, U.: Zur Föderalismusreform in Deutschland, in: Juristenzeitung, 61/19 (2006), 930-940, hier 931. 
mit einem Frühwarnsystem sowie die Etablierung verursachungsgerechter Sanktionsmechanismen nach Vorbild des EU-Stabilitätspaktes. Derartigen Forderungen liegt die Überzeugung zugrunde, dass jedes Bundesland für seine finanziellen Geschicke künftig mehr Verantwortung übernehmen soll.

Eine wesentliche Voraussetzung dieses Vorhabens ist eine hinreichende Handlungsautonomie der Bundesländer in Form von finanzwirksamen Gestaltungsund Innovationsspielräumen. Diese stellen gleichsam ein „Ventil“ für den Druck dar, der aus einer verfassungsrechtlichen Verschuldensgrenze samt Sanktionsmechanismen für die Länder (und Kommunen) notwendig folgt. Hinzu muss die Garantie kommen, etwaige Einsparungen zur Konsolidierung des Länderhaushalts oder für neuerliche Investitionen behalten zu dürfen. Nur unter diesen Voraussetzungen bestehen Anreize für ein Bundesland, seine Ausgaben zu minimieren und die preiswerteste Alternative der Aufgabenerfüllung zu ermitteln.

Um aber zu vermeiden, dass der Begriff des „Föderalismus“ zu einem Spielball des politischen Prozesses, der je nach Bedarf zur Begründung unterschiedlichster Interessen eingesetzt wird - heute dafür, morgen dagegen - bedarf es eines die Thematik überwölbenden Leitbildes für das Verständnis unseres Bundesstaates und des in ihm gelebten Föderalismus. Denn ein Föderalismus, dessen Funktion unklar ist, ist seiner schleichenden Aushöhlung schutzlos ausgeliefert. Nur wenn Klarheit und Konsens über den Sinn und Zweck des bundesdeutschen Föderalismus besteht, mithin ein klares Leitbild des Föderalismus besteht, können im Bund-Länder Verhältnis aufgeworfene Probleme und Rechtsfragen in sich schlüssig beantwortet werden. Bildlich gesprochen geht es daher darum, nicht nur die einzelnen Bäume anzuschauen, sondern auch einmal den ganzen Wald aus der Vogelperspektive in den Blick zu nehmen.

\section{Ein neues Leitbild: Innovationsföderalismus}

\section{Grundlagen}

Auch wenn das Grundgesetz von Anfang an Verflechtungen und Kooperationsformen, etwa beim Vollzug von Bundesgesetzen oder in der Gesetzgebung durch die Institution des Bundesrates, zwischen den beiden Ebenen des Bundesstaates vorsah, so war sein Grundmuster doch von einem Trennsystem geprägt, das eher 
dem Modell des dualistischen Föderalismus entsprach. ${ }^{3}$ Unter dem Druck der Verhältnisse setzte jedoch schon bald eine schleichende Umwandlung vom die Länderstaatlichkeit betonenden dualistischen zum sog. unitarisch-kooperativen Föderalismus ein.

Abgesehen davon, dass die traditionelle Verwurzelung in landsmannschaftlichen und kulturellen Eigenarten durch die Folgen des Zweiten Weltkrieges nach 1945 verwischt worden war und die Bildung der Bundesländer zumeist nicht an historisch vorgegebene staatliche Einheiten anknüpfte, waren die moderne Industriegesellschaft und das Sozialstaatsprinzip mit ihren Vereinheitlichungsbedürfnissen für die Entwicklung hin zum sog. unitarisch-kooperativen Föderalismus verantwortlich. In diesem Kontext hat sich der bundesdeutsche Föderalismus von seiner ihm historisch eigentlich zukommenden Funktion, durch „Wahrung der Vielfalt in der Einheit" die Existenz gegenläufiger integrativer und desintegrativer politisch-kultureller Kräfte im geographischen Raum des Territorialstaates auszugleichen, weitgehend gelöst. ${ }^{4}$ Bildlich gesprochen ist der Bundesstaat der Verfassungsväter mit seinen klaren Abgrenzungen in Form einer "Schichttorte“ im Zuge dieser Entwicklung dem kooperativen Föderalismus in Form eines "Marmorkuchens" gewichen.

Nachdem dieser kooperative Föderalismus zunächst als dynamische Fortentwicklung, die alle bundesweiten Probleme im Infrastrukturbereich bzw. der Wirtschafts- und Sozialvorsorge lösen könne, begrüßt worden war, ${ }^{6}$ wird er heute in der politischen Praxis ebenso wie in der wissenschaftlichen Literatur überwiegend negativ beurteilt. ${ }^{7} \mathrm{Zu}$ Recht wurden als nachteilige Folgen immer wieder (1) die mangelnde Effektivität als Folge der Notwendigkeit einstimmiger Entscheidungen, die - angesichts von Gleichbehandlung und Besitzstandswahrung der Länder - nur einen Konsens auf dem kleinsten gemeinsamen Nenner ermöglichen, (2) die Bürokratisierung der Willensbildungs- und Entscheidungsprozesse in administrativen Gremien (sog. Exekutivföderalismus), (3) die dop-

3 So die h.M., vgl. nur Ossenbühl, F.: Landesbericht Bundesrepublik Deutschland, in: ders. (Hg.): Föderalismus und Regionalismus in Europa, Baden-Baden, 1990, 117-165, hier $151 \mathrm{f}$.; Grimm, D.: Die Zukunft der Verfassung, Frankfurt/M., 1991, 348 ff.; a. A. Stern, K.: Das Staatsrecht der Bundesrepublik Deutschland, Bd. I, 2. Aufl., München, 1984, 754: „tendentiell kooperativ“.

4 Anderer Auffassung Isensee, J.: Idee und Gestalt des Föderalismus im Grundgesetz, in: ders./Kirchhof, P. (Hg.): Handbuch des Staatsrechts, Heidelberg, 1990, § 98, Rn. 305 ff.

5 Klatt, H.: Reform und Perspektiven des Föderalismus in der Bundesrepublik Deutschland: Stärkung der Länder als Modernisierungskonzept, in: Aus Politik und Zeitgeschichte, B28/1986, 3-21, hier 8.

6 Hesse, K.: Der unitarische Bundesstaat, Karlsruhe, 1962, $27 \mathrm{f}$.

7 Vgl. hier nur Klatt, H., a. a. O., 8. 
pelte Entmachtung der Länderparlamente durch den Verlust an Entscheidungsmacht und Gesetzgebungskompetenzen, (4) die angesichts mangelnder Transparenz der Willensbildung und diffuser politischer Verantwortung entstehenden Kontrolldefizite sowie (5) der generelle Verlust an Gestaltungsspielraum und damit Staatlichkeit der Länder beklagt.

\section{Das neue perspektivische Leitbild des Innovationsföderalismus}

Vor diesem Hintergrund bedarf der Föderalismus in der Bundesrepublik ${ }^{8}$ einer neuen Legitimationsgrundlage. Sucht man nach einer erneuerten Legitimationsgrundlage, so stößt man auf das dem Föderalismus immanente Potential der Gleichzeitigkeit mehrerer Entscheidungsebenen. Insoweit rückt die innovationsfördernde Funktion des Föderalismus im Verhältnis der Länder untereinander, aber auch gegenüber dem Bund in das Zentrum des Interesses. Stichworte sind insoweit die Ermöglichung von Experimenten in kleinerem Rahmen, die Bürgernähe sowie die Verbreiterung von Mitwirkungschancen. ${ }^{9}$

Diese Funktion wird auch unter dem Stichwort „Konkurrenzföderalismus“10 oder „kompetitiver Föderalismus“"11 beschrieben, gleichwohl beschreibt der konstruktive Begriff der „Gestaltung“ bzw. der „Innovation“ treffender worum es eigentlich geht.

Innovationsföderalismus lässt sich im System der Bundesrepublik dahingehend verstehen, dass im Rahmen der bundesstaatlichen Ordnung des Grundgesetzes zwischen Bund und Ländern sowie zwischen den Länder untereinander innovationsfördernde Gestaltungsspielräume sowohl bei der Lösung von politischen Problemen als auch bei der Wahrnehmung staatlicher Aufgaben ermöglicht werden. $^{12}$

Damit ist die Frage aufgeworfen, woraus sich der so beschriebene Grundgedanke des Innovationsföderalismus legitimiert. In Westeuropa lassen sich gegenwärtig

8 Dazu Bothe, M.: Föderalismus - ein Konzept im geschichtlichen Wandel, in: Evers, T. (Hg.): Chancen des Föderalismus in Deutschland und Europa, Baden-Baden, 1994, S. 19-32, hier 21 m. w. N.

9 Vgl. dazu den Überblick bei Bothe, ebd., 24 ff.; Isensee, J., a. a. O., Rn. 299 ff.

10 Vgl. dazu Klatt, H.: Parlamentarisches System und bundesstaatliche Ordnung: Konkurrenzföderalismus als Alternative zum kooperativen Bundesstaat, in: Aus Politik und Zeitgeschichte, B31/1982, 3-24, hier $21 \mathrm{ff}$.

11 Calliess, C.: Die Justitiabilität des Art. 72 Abs. 2 GG vor dem Hintergrund von kooperativem und kompetitivem Föderalismus, in: Die öffentliche Verwaltung, 50/21 (1997), 889-899 m.w. N.

12 Ähnlich Klatt, H.: Parlamentarisches System, a. a.O., 22. 
zwei unterschiedliche Entwicklungen feststellen. Deren eine begünstigt supranationale Strukturen, um in Zeiten wachsender Verflechtung und Abhängigkeiten (Globalisierung) eine problemadäquate Politikebene zu etablieren. Deren andere begünstigt dezentrale Strukturen unterhalb der nationalstaatlichen. Zum Stichwort ist hier das „Europa der Regionen“ geworden. ${ }^{13}$ Letzterer Trend entspringt zum Teil der Suche nach einer Kompensation für den kulturellen Identitätsverlust, der in der Konsequenz der zunehmenden Globalisierung und Bildung supranationaler Strukturen und der damit einhergehenden interdependenten Kommunikationsgesellschaft liegt. ${ }^{14}$

Von Bedeutung ist aber noch ein weiterer - im Folgenden als „Gouvernanzoptimierung" bezeichneter - Aspekt, nämlich die wachsende Einsicht, dass staatliche Politik in einer komplexen, von qualitativ hochwertigen Informationen abhängigen modernen Industriegesellschaft dezentraler politischer Handlungsebenen bedarf, um problemlösungsfähig zu bleiben. ${ }^{15} \mathrm{Zu}$ Recht wird insofern betont, dass dezentrale Lösungen die Komplexität des Entscheidungsprozesses verringern und zu einer erhöhten Transparenz beitragen. Die betroffenen Menschen können sich besser mit den politischen Entscheidungen identifizieren, wenn diese bürgernah getroffen werden. Dies nicht zuletzt deshalb, weil dezentrale Entscheidungen eine stärkere Partizipation und damit ein Mehr an Demokratie ermöglichen können. Indem politische Lösungen auf dezentralen Ebenen sich dem konkreten Problem vor Ort besser anpassen können, wird die Effizienz des gesamten Entscheidungsprozesses verbessert. ${ }^{16}$ Auf Informationsdefiziten beruhende Irrtümer zentraler Entscheidungsebenen können dadurch vermieden werden, dass Entscheidungen dort getroffen werden, wo die Informationen angesiedelt sind. ${ }^{17}$

Die Mehrstufigkeit eines politischen Systems mit dezentralen Handlungsebenen bewahrt nicht zuletzt soziale, kulturelle, wirtschaftliche und politische Vielfalt und reflektiert damit die verschiedenen Wünsche, Einstellungen und Bedürfnisse

13 Stammen, T.: Das Phänomen des europäischen Regionalismus, in: Kremer, H. A. (Hg.): Die Landesparlamente im Spannungsfeld zwischen europäischer Integration und europäischem Regionalismus, München, 1988, 163 ff.; Häberle, P.: Der Regionalismus als werdendes Strukturprinzip des Verfassungsstaates und als europarechtspolitische Maxime, in: Archiv des öffentlichen Rechts, 118/1 (1993), 1-44.

14 Vgl. Lübbe, H.: Die große und die kleine Welt. Regionalismus als europäische Bewegung, in: Weidenfeld, W. (Hg.): Die Identität Europas, Bonn, 1985, 191-205; Stammen, T., a. a. O., $166 \mathrm{ff.}$

15 Vgl. Beck, U.: Risikogesellschaft, Frankfurt/M., 1986, $311 \mathrm{ff}$.

16 Vgl. Mayntz, R.: Föderalismus und die Gesellschaft der Gegenwart, in: Archiv des öffentlichen Rechts, 115/2 (1990), 232-245, hier 235, 239.

17 Vgl. hierzu Beck, U., a. a. O., 369. 
der Bürger. Diese Vielfalt erlaubt Spielraum für Experimente bei der Lösung von Problemen, deren Erfahrungen dann dem Gesamtsystem zugute kommen könnten. ${ }^{18}$ Wo Handlungs- und Entscheidungsspielräume für dezentrale Ebenen eröffnet werden, werden die Vorteile des Wettbewerbs für ein politisches System genutzt. ${ }^{19}$ Jene Spielräume tragen auf diese Weise zu einer Verbesserung der Effizienz des Gesamtsystems bei. ${ }^{20}$ Überdies bestehen länderspezifische Rahmenbedingungen, die entweder mildernd oder intensivierend wirken können, so dass nicht nur der Problemlösungsbedarf und der Problemlösungsdruck räumlich variieren, sondern sich auch je nach den lokalen Gegebenheiten recht unterschiedliche Problemlösungsstrategien anbieten. Dieser Effizienzvorteil eines mehrstufigen Systems kann sich aber nur entfalten, wenn einerseits die Frage der vertikalen Aufgabenteilung (Subsidiarität) und andererseits die Frage der vertikalen und horizontalen Verflechtung (Solidarität) gelöst werden. ${ }^{21}$

Einen ersten vorsichtigen Schritt dahingehend hat die „kleine“ Föderalismusreform von 1994 mit dem 42. Änderungsgesetz unternommen, indem sie im Bereich konkurrierender Gesetzgebungszuständigkeiten die sog. „Bedürfnisklausel“ des Art. 72 Abs. 2 GG durch eine „Erforderlichkeitsklausel“ ersetzte, mit dem Ziel die Inanspruchnahme der Titel des Art. 74 GG durch den Bund zu erschweren und den Länder Gestaltungsspielräume - und damit auch Innovationsspielräume - zu sichern. Die Neuformulierung sollte die Darlegungslast des Bundes erhöhen und die Norm justiziabler machen (vgl. auch die zeitgleich neu eingeführte Zuständigkeit des BVerfG in Art. 93 Abs. 1 Nr. 2a GG). Trotz vielfältiger Zweifel in der Literatur ${ }^{22}$ hat das BVerfG mit seiner Altenpflege-Entscheidung ${ }^{23}$ den ihm solchermaßen zugespielten Ball angenommen und die Inanspruchnahme einer konkurrierenden Zuständigkeit durch den Bund erstmals am Maßstab des Art. 72 Abs. GG genau überprüft.

Einen weiteren Schritt unternahm die Föderalismusreform I von 2006, indem sie - mit dem Ziel einer Entflechtung von Zuständigkeiten - die Rechtsetzungskompetenzen neu verteilte, insbesondere die Rahmengesetzgebungskompetenz

18 Gretschmann, K.: The Subsidiarity Principle, in: Institut Européen d'Administration Publique (Hg.): Subsidiarité, Maastricht, 1991, $7 \mathrm{ff}$.

19 Ebd., 8.

20 Vgl. Mayntz, R., a. a. O., 235, 239.

21 Ebd., 241 f. m.w. N.

22 Dagegen Calliess, C., a. a. O., m.w. N.

23 BVerfGE 109, 190 ff., 229 ff.; hierzu Calliess, C.: Kontrolle zentraler Kompetenzausübung in Deutschland und Europa, in: Europäische Grundrechtezeitschrift, 30/7-10 (2003), 181-197. 
des Bundes abschaffte und dafür eine gestaltungs- und innovationsfreundliche Abweichungsgesetzgebungskompetenz der Länder in Art. 72 Abs. 3 GG etablierte sowie die Zahl der von einer Zustimmung des Bundesrates abhängigen Gesetze reduzierte.

Das System des Innovationsföderalismus setzt aber überdies eine Finanzverfassung voraus, die geeignet ist, politische Abhängigkeiten und rechtliche Unsicherheiten zu beseitigen und gesicherte Rahmenbedingungen für die einem föderalem System eigenen und erforderlichen Auseinandersetzungen zwischen dem Zentralstaat und den Gliedstaaten zu schaffen. ${ }^{24}$ Ohne eine hinreichende Finanzausstattung der Länder besteht die latente Gefahr, dass jedwede Innovationsbereitschaft im Wege einer „Politik des goldenen Zügels“ bereits im Keim erstickt wird. Ein solcher Innovationsföderalismus über trial and error ist demgegenüber insbesondere in den USA immer wieder wirksam geworden: Einzelstaaten sind bei bedeutenden neu auftauchenden Bedürfnissen oftmals in der Gesetzgebung vorgeprescht. Teils wurde damit Bundesgesetzgebung eingeleitet, teils die Möglichkeit der befriedigenden Regelung durch einzelstaatliches Recht bestätigt. ${ }^{25}$ Auch ein bundesweiter Innovationswettbewerb - verstanden als Verfahren zur Entdeckung der besten Problemlösung ${ }^{26}$ - wird sich nur auf Basis der obigen Vorgaben ergeben. Das Erfordernis einer hinreichenden Eigenverantwortlichkeit der Länder ist somit conditio sine qua non dafür, dass die ureigensten Stärken des Bundesstaates wieder aufleben. Denn ohne derartige Freiräume fehlt jede Möglichkeit zur experimentellen Erprobung bzw. Generierung alternativer Lösungswege, so dass die positiven Effekte der kulturellen, wirtschaftlichen und politischen Vielfalt auf Länderebene ungenutzt bleiben. ${ }^{27}$

Vor diesem Hintergrund sollen nachfolgend Überlegungen angestellt werden, wie sich finanzwirksame Innovations- und Gestaltungsspielräume der Länder mit dem Ziel herstellen lassen, eigenverantwortliche Einsparungen auf Länderebene zu ermöglichen.

24 Ausführlich Lensch, R.: Die Finanzverfassung des Grundgesetzes und die neuen Länder, in: Evers, T. (Hg.): Chancen des Föderalismus in Deutschland und Europa, Baden-Baden, 1994, 95 ff., hier insbesondere $114 \mathrm{f}$; ähnlich BVerfGE 72, $330 \mathrm{ff}$., hier $388 \mathrm{f}$.

25 Hierzu ausführlich Frowein, J. A.: Konkurrierende Zuständigkeit und Subsidiarität. Zur Kompetenzverteilung in bündischen Systemen, in: Badura, P./Scholz, R. (Hg.): Wege und Verfahren des Verfassungslebens. Festschrift für Peter Lerche, München, 1993, 401-410, hier $403 \mathrm{ff}$., $408 \mathrm{f}$.

26 Näher dazu Hayek, F. A.: Der Wettbewerb als Entdeckungsverfahren, Kiel, 1968, 14.

27 Mayntz, R., a.a.O., 235 f.; Calliess, C.: Die Justitiabilität des Art. 72 Abs. 2 GG, a.a.O., 891 f.; Mammen, L.: Der neue Typus der konkurrierenden Gesetzgebung mit Abweichungsrecht, in: Die öffentliche Verwaltung, 60/9 (2007), 376-380, hier 379. 


\section{Aufgabenoptimierung durch Koordinierung und benchmarking}

Unter dieser Überschrift stellt sich die Frage, ob und inwieweit dadurch finanzierungsrelevante Gestaltungs- und Innovationsspielräume dadurch geschaffen werden können, dass der Bund im Interesse gleichartiger Regelungen im Bundesgebiet zwar koordinierend tätig wird, den Länder aber nur Ziele vorgibt, deren Verwirklichung im Rahmen eines benchmarking dann verglichen und überprüft werden kann.

\section{Zum Verständnis einer etwaigen Koordinierungszuständigkeit des Bundes}

In der aktuellen Debatte ist des Öfteren von einer Koordinierungszuständigkeit des Bundes die Rede. So gibt es Überlegungen des Bundes, im Bereich des Katastrophen- und Zivilschutzes sowie im IT-Bereich eine solche neue Zuständigkeit zu begründen. Schaut man sich freilich die Vorstellungen an, die der Bund von einer solchen Koordinierungszuständigkeit hat, wird deutlich, dass der Begriff der Koordinierung unklar ist, die Bundeszuständigkeit insoweit also über das eigentliche Ziel schnell hinausgehen könnte. Bei aller Einsicht in die sachliche Notwendigkeit einer Koordinationsrolle des Bundes in den genannten Bereichen ist der Begriff der Koordinierung und damit auch derjenige der Koordinierungszuständigkeit zunächst einmal inhaltlich zu präzisieren. Andernfalls könnte sich der zunächst harmlos klingende Begriff der „Koordinierungszuständigkeit“ in den genannten Bereichen dann schnell als „Falschetikettierung“ erweisen, im Zuge derer dem Bund, entgegen dem Leitbild des Innovationsföderalismus, eine nicht sachdienliche Vereinheitlichungszuständigkeit zuwachen könnte.

Der Begriff der Koordinierung ist aus dem Europarecht bekannt. Es bietet sich daher an, ihn von dort aus kommend im Lichte des Innovationsföderalismus zu definieren. Er beschreibt hier unter der Bezeichnung Offene Methode der Koordinierung (OMK) zunächst einmal einen governance-Modus im Rahmen der allgemeinen EU-Wirtschaftspolitik (Art. 99 Abs. 1 EGV) zwischen den Mitgliedstaaten. Insoweit geht es um die Abstimmung der weitgehend in der Hand der Mitgliedstaaten verbliebenen allgemeinen Wirtschaftspolitik, die an den Vorgaben der Wirtschaftpolitik nach Art. 99 Abs. 2 Satz 3 i.V.m. 98 Abs. 1 EGV ausgerichtet werden muss. Konkrete Anwendungsgebiete der OMK fanden sich zunächst im Bereich der Schwerpunkte der sog. Lissabon-Strategie, der 
Arbeitsmarkt- und Sozialpolitik. ${ }^{28}$ Insoweit sollte die OMK Bereiche erfassen, die teilweise außerhalb der Kompetenz der EU liegen.

Bei der OMK werden die Mitgliedstaaten also in Sachbereichen, in denen die Union eigentlich keine Zuständigkeiten besitzt, über eine schrittweise Entwicklung und gegenseitige Anpassung ihrer nationalen Politiken aufgrund gewisser Vorgaben der Union zu einem gemeinsamen - koordinierten - Vorgehen im Rahmen des Europäischen Rates veranlasst. ${ }^{29}$ Die von der Union vorgegebenen Leitlinien bzw. Ziele, ${ }^{30}$ an denen sich die Mitgliedstaaten bei der Ausgestaltung ihrer nationalen Politiken orientieren sollen, sind dabei rechtlich unverbindlich. Die Entscheidung über Intensität und Ausmaß der Anpassung ihrer nationalen Politiken bleibt - sieht man von dem ,faktischen Handlungsdruck“, der nach Auffassung im Schrifttum im Rahmen dieser Methode wohl entsteht, ${ }^{31} a b$ - den Mitgliedstaaten überlassen.

Konkret werden im Rahmen der OMK gemeinsamer Ziele auf EU-Ebene mit einem genauen Zeitplan, sowie die Festlegung von Indikatoren und benchmarks definiert. Es folgt die freiwillige Unterwerfung der Mitgliedstaaten unter diese Ziele und die Umsetzung der Ziele durch regional spezifische Maßnahmen. Die Überwachung der Zielerreichung oder wenigstens der Fortschritte erfolgt auf der Basis jährlich zu erstellender Aktionspläne im Rahmen eines peer-reviewVerfahrens, das durch den Europäischen Rat durchgeführt wird. Dieses Verfahren ist methodisch als ein qualitativer Evaluationsprozess zu verstehen.

Die OMK ist kein Rechtsetzungsverfahren, selbst wenn sie in ein gesetzgebendes Rahmenwerk eingebunden ist und gegebenenfalls sogar in konkrete Rechtsetzung mündet. Vielmehr geht es um die Unterstützung nationaler Politikformulierung und ihre Koordinierung durch eine Verständigung auf gemeinsame Ziele

28 Europäischer Rat (Lissabon, 23./24.03. 2000): Schlussfolgerungen des Vorsitzes, BullEU 3/2000, Ziff. I.5.5.7.

29 Vgl. ausführlich: Bodewig, T./Voß, T.: Die „offene Methode der Koordinierung“ in der Europäischen Union - ,schleichende Harmonisierung“ oder notwendige „Konsentierung“ zur Erreichung der Ziele der EU, in: Europarecht, 38/2 (2003), 310-326; de Búrca, G.: The constitutional challenge of new governance in the European Union, in: European Law Review, 28/6 (2003), 814-839.

$30 \mathrm{Zu}$ den einzelnen Vorgaben: Europäischer Rat (Lissabon, 23./24. 03. 2000), a. a. O., Ziff. I.18.37 ff.

31 Nettesheim, M.: Die Kompetenzordnung im Vertrag über eine Verfassung für Europa, in: Europarecht, 39/4 (2004), 511-546, hier 531; vgl. auch: Schwarze, J.: Der Verfassungsentwurf des Konvents - Struktur, Kernelemente und Verwirklichungschancen, in: ders. (Hg.): Der Verfassungsentwurf des Europäischen Konvents, Baden-Baden, 2004, 489-564, hier 520; ders.: Das wirtschaftsverfassungsrechtliche Konzept des Verfassungsentwurfs des Europäischen Konvents, in: Europäische Zeitschrift für Wirtschaftsrecht, 15/5 (2004), 135-140, hier 138, spricht von der Gefahr einer ,vorprägenden Quasi-Gesetzgebungsmacht". 
und Umsetzungsmaßnahmen. Im Rahmen eines Austausches von Informationen (vgl. Art. 99 Abs. 2 Satz 3 EGV) sollen Lernprozesse sowohl in den Regierungen der Mitgliedstaaten als auch in den nationalen Parlamenten initiiert werden, die langfristig in transnationalen Lernnetzwerken resultieren. Der Anreiz, die besten Konzepte und Instrumente innerhalb der EU-Mitgliedstaaten $\mathrm{zu}$ entwickeln, basiert unter anderem darauf, dass ,als Belohnung“ die Vergemeinschaftung des eigenen Steuerungsansatzes in der Europäischen Union winkt. So werden Anpassungskosten in dem Mitgliedstaat vermieden, dessen Konzept sich durchsetzt.

Angelehnt an diese Konzeption, die weitgehend außerhalb der Verträge entstanden ist, hat der von allen Mitgliedstaaten unterzeichnete, aber dann doch nicht ratifizierte Verfassungsvertrag die Koordinierung als eigenständige Kompetenzkategorie der EU eingeführt, der Vertrag von Lissabon hat die Regelungen übernommen.

In Anlehnung an dieses europarechtlich vorgeformte Verständnis kann Koordinierung im Bund-Länder-Verhältnis nur eine begrenzte Kompetenz des Bundes bedeuten, als deren Kehrseite das Gros einer Zuständigkeit bei den Ländern bleibt. Sinn und Zweck ist eine schrittweise Entwicklung und gegenseitige Anpassung der Länderpolitiken an die Vorgaben des Bundes. Insoweit darf der Bund nur unterstützend tätig werden. Unterstützend heißt, der Bund ist auf die Handlungsform der Koordinierung beschränkt. Diese erlaubt nur Vorgaben in Form von Zielen und Leitlinien, an denen sich die Länder bei der konkreten Ausgestaltung ihrer Politiken verpflichtend auszurichten haben. Die regionalen Unterschiede und Lösungsansätze in den Ländern sollen sodann genutzt werden, um nach best practice-Modellen zu suchen: Insoweit ermöglichen Instrumente wie benchmarking und peer review Vergleiche zwischen den Ländern, im Zuge derer dann ein entsprechender policy-Transfer im Hinblick auf das Ländermodell, das die vorgegebenen Ziele am besten umsetzt, erfolgen kann.

Im Rahmen einer so verstandenen Kombination aus Koordinierung und benchmarking könnten den Ländern Gestaltungs- und Innovationsspielräume zuwachsen. Eine so verstandene Koordinierungszuständigkeit des Bundes wäre - Subsidiaritäts- und Verhältnismäßigkeitsprinzip korrespondierend - ein milderes Mittel im Vergleich zur Vollgesetzgebung aufgrund konkurrierender Bundeszuständigkeit. 


\section{Konkrete Ausgestaltung eines Länder-benchmarking}

Das vornehmlich aus der Ökonomie bekannte, aber auch schon auf kommunaler Ebene eingesetzte Flexibilisierungsmitte ${ }^{32}$ des benchmarking definiert sich dadurch, dass den Ländern standardisierte Ziele, Leitlinien oder Kennzahlen vom Bund als koordinierender Ebene vorgegeben werden. Dabei handelt es sich um verbindliche Zielsetzungen in Bezug auf das zu erreichende Ergebnis, während die Ausgestaltung des einzuschlagenden Weges frei von etwaigen Weisungen bleibt, so dass den Länder insoweit Gestaltungs- und Innovationsspielräume zukommen. Diese Autonomie ermöglicht die Ermittlung sog. best practices, weil man die Leistungen und Ergebnisse der zur Umsetzung verpflichteten Länder vergleichen und dadurch die besten Lösungen generieren kann. Länder mit ,unterlegenen" Varianten haben infolgedessen die Möglichkeit, bessere Versionen anderer Länder im Falle ihrer Übertragbarkeit zu kopieren und können so aus etwa gemachten Fehlern lernen. ${ }^{33}$

Auf Basis dieser Grobbeschreibung ergeben sich die einzelnen Stadien eines „Gesetzes-benchmarking“" wie folgt: ${ }^{34}$

- Zunächst muss der Bund in quantitativer und qualitativer Hinsicht leistungsfähige Kennzahlen ermitteln. Dazu bedarf es der Auswahl solcher Gesetzesmaterien, die alle (zur Teilnahme verpflichteten) Länder gleichermaßen betreffen, und einer hinreichend exakten Festsetzung des zu erreichenden Leistungsniveaus. Die Vorgabe sollte zudem im Bereich des Machbaren liegen, um die Wettbewerber nicht durch das Gefühl ,anfänglicher Unmöglichkeit“ zu demotivieren. Ferner bedarf es aussagefähiger Vergleichsparameter - in der Regel der detailliert zu quantifizierende Vollzugsaufwand des Gesetzes in personeller und sachlicher Hinsicht; denn sonst wären die unterschiedlichen Gesetze keiner Bewertung zugänglich.

- Im Anschluss daran sind die Länder gefordert, diesen Kennzahlen innerhalb der nunmehr zu konzipierenden Gesetze zu entsprechen. Dieser Prozess hat ohne länderübergreifende Abstimmung zu erfolgen, um das Innovationspo-

32 Vgl. dazu etwa Budäus, D./Finger, S.: Stand und Perspektiven der Verwaltungsreform in Deutschland, in: Die Verwaltung, 32/3 (1999), 313-343, hier $313 \mathrm{ff}$.

33 Machura, S.: Leistungsmessung in der öffentlichen Verwaltung, in: Die Verwaltung, 32/3 (1999), 403427, hier 417 ff.; Budäus, D./Finger, S., a. a. O., 323.

34 Vgl. dazu Kuhlmann, S.: Benchmarking auf dem Prüfstand: Kosten, Nutzen und Wirkungen interkommunaler Leistungsvergleiche in Deutschland, in: Verwaltungsarchiv, 94/1 (2003), 99-126, hier 103, jedoch zum interkommunalen Leistungsvergleich. 
tenzial eines Gesetzes-benchmarking voll auszuschöpfen und Trittbrettfahrerverhalten ${ }^{35} \mathrm{zu}$ vermeiden.

- Nach Verabschiedung der Gesetze und nach Abschluss des Erhebungszeitraums ist zum einen zu prüfen, ob die Kennzahlen eingehalten worden sind. Sollte dies nicht der Fall sein, sind dem Bund Möglichkeiten an die Hand zu geben, um die von ihm gesetzten Kennzahlen z. B. durch gesetzliche Anweisungen o. ä. durchzusetzen. Zum anderen ist im Wege der Interpretation des gesetzesdeterminierten Vollzugsaufwandes zu ergründen, welches Bundesland den effizientesten und effektivsten Rechtsakt konzipiert hat, wobei es einer substantiierten Darlegung der entstandenen Kosten bedarf, weil sonst ein Vergleich schon dem Grunde nach scheitern wird. ${ }^{36}$

- Abschließend muss ggf. unter Federführung des Bundes ${ }^{37}$ ergründet werden, warum bestimmte Landesgesetze anderen in Bezug auf die verursachten Kosten unterlegen sind und inwieweit die best practices übertragen werden können ${ }^{38}$. Bejahendenfalls ist die überlegene Lösung in das Recht der anderen Länder zu implementieren, wobei dem Bund ein Weisungsrecht zukommen könnte, um dem „Gesetzes-benchmarking“ hinreichend Verbindlichkeit zu verschaffen. Ein weiteres disziplinierendes Moment kann sich indes durch eine bundesweite Veröffentlichung der jeweiligen Zielerreichungsgrade der einzelnen Länder erreichen lassen, um den mündigen Bürger als weitere Kontrollinstanz zu aktivieren. ${ }^{39}$

\section{Verfassungsrechtliche Zulässigkeit des skizzierten Länder- benchmarkings}

Insbesondere mit Blick auf die vorstehend skizzierten Zwangsmittel stellt sich allerdings die Frage nach der verfassungsrechtlichen Zulässigkeit eines benchmarking-Systems.

35 So das Verhältnis der südafrikanischen zur namibischen Gesetzgebung nach Woite, J.: Gesetzesfolgenabschätzung - Modeerscheinung oder Notwendigkeit, in: Zeitschrift für Rechtspolitik, 33/6 (2000), 260.

36 So auch (zur prospektiven Gesetzesfolgenabschätzung) Scholz, R./Meyer-Teschendorf, K. G.: Reduzierung der Normenflut durch qualifizierte Bedürfnisprüfung, in: Zeitschrift für Rechtspolitik, 29/10 (1996), 404-408, hier 406.

37 Insoweit weicht die hier vorgeschlagene Methode von der auf kommunaler Ebene angewandten ab, vgl. dazu Machura, S., a. a. O., 418 f., 421.

38 Dieses Erfordernis befürwortet auch Woite, J., a. a. O.

39 Vgl. dazu näher Calliess, C./Korte, S.: Das neue Recht der Grünen Gentechnik im europäischen Verwaltungsverbund: zur Verzahnung staatlicher und europäischer Risikovorsorge im Kontext von Regulierung und Selbstregulierung, in: Die öffentliche Verwaltung, 59/1 (2006), 10-22, hier $21 \mathrm{f}$. 


\section{a) Formelle Grenzen}

Ein Gesetzes-benchmarking ist sinnvollerweise rechtsgebietsbezogen auszugestalten. Der in diesem Zusammenhang erforderliche Spielraum für die Landesgesetzgebung lässt sich im Anwendungsbereich einer ausschließlichen Rechtsetzungskompetenz nach Art. 73 GG allerdings nur herstellen, wenn der Bund die Länder im Sinne des Art. 71 GG ausdrücklich zur Gesetzgebung ermächtigt. ${ }^{40}$ Da Art. 71 GG jedoch als Ausnahmevorschrift konzipiert ist, darf der Bund eine ihm zugewiesene Kompetenz nicht in Gänze aufgeben und muss für eine Ermächtigung an die Länder einen sachlichen Grund vorweisen. Zudem ist ihm eine substanzielle Verlagerung von Zuständigkeiten versagt, weil sonst das in den Art. $70 \mathrm{ff}$. GG angelegte Kompetenzgefüge durchbrochen wäre. ${ }^{41}$ Diese engen Grenzen führen dazu, dass ein Gesetzes-benchmarking im Anwendungsbereich der Art. 71, 73 GG nur in seltenen Fällen möglich sein dürfte - so z. B. wenn der Bund den Ländern die Festlegung der konkreten Mittel zum Schutz deutschen Kulturguts gegen Abwanderung ins Ausland (vgl. Art. 73 Abs. 1 Nr. $5 \mathrm{a} \mathrm{GG)} \mathrm{überlässt.}$

Hauptsächlich könnte ein Gesetzes-benchmarking somit im Bereich konkurrierender Rechtsetzungszuständigkeiten Anwendung finden, wobei die in Art. 74 Abs. 1 GG niedergelegten Kompetenztitel ein derartiges Mittel zur innovationsorientierten Gesetzgebungsoptimierung auch tragen. So ist zum einen die Vorgabe von Kennzahlen unproblematisch möglich, weil der Bund allein durch die Niederlegung von Wert- und Zielvorstellungen noch keinen Gebrauch von seinen Gesetzgebungskompetenzen im Sinne des Art. 72 Abs. 1 GG macht. ${ }^{42}$ Daher ist er auch befugt, den Landesgesetzgebern im Bereich der konkurrierenden Zuständigkeiten lediglich Rahmenvorgaben zu machen. ${ }^{43}$ Zum anderen erfassen die in Art. 74 GG aufgeführten Kompetenztitel sowohl materielle als auch verfahrensrechtliche Fragen, so dass auch die für ein funktionstüchtiges Gesetzesbenchmarking erforderlichen Weisungsrechte des Bundes gegenüber den Ländern unter die dortigen Katalogtatbestände fallen. ${ }^{44}$ Dafür spricht insbesondere, dass der Bund im Falle des Gesetzes-benchmarking von den ihm möglichen

40 Sannwald, R., in: Schmidt-Bleibtreu, B./Klein, F. (Hg.): Kommentar zum Grundgesetz, 10. Aufl., Neuwied, 2004, Art. 71, Rn. 9.

41 Degenhart, C., in: Sachs, M. (Hg.): Grundgesetz: Kommentar, 4. Aufl., München, 2007, Art. 71, Rn. 10.

42 Sannwald, R., a. a. O., Art. 72, Rn. 30; BVerfGE 34, 9 ff., hier 28.

43 Herzog, R., in: Maunz, T./Dürig, G. (Begr.): Grundgesetz-Kommentar, Bd. 5, München, 23. EL 1984, Art. 72, Rn. 13.

44 So andeutungsweise auch Degenhart, C., a.a. O., Art. 74, Rn. 3. 
Rechtsetzungsbefugnissen einen Teil auf die Länder überträgt, indem er sich auf die Normierung von Kennzahlen und Kontrollmechanismen beschränkt. Dadurch entspricht er dem in Art. 70 GG niedergelegten, dem Subsidiaritätsprinzip korrespondierenden Grundsatz der vorrangigen Länderzuständigkeit eher als durch eine materielle Vollregelung, so dass ihm ein rechtsgebietsbezogenes Gesetzesbenchmarking auf Basis der Art. 74, 72 GG erst recht (a majore ad minus) möglich sein muss.

\section{b) Materielle Grenzen}

Fraglich bleiben somit nur noch die materiellen Grenzen des Gesetzes-benchmarking.

\section{aa) Allgemeiner Gleichheitssatz}

In diesem Zusammenhang ist zunächst eine Verletzung des allgemeinen Gleichheitssatzes zu prüfen, der entweder als objektives Willkürverbot oder als allgemeiner rechtsstaatlicher Grundsatz auch im Bund-Länder-Verhältnis gilt. ${ }^{45} \mathrm{Er}$ verbietet nicht nur, ohne sachlichen Grund ungleich, sondern vielmehr auch umgekehrt ungleiches ohne sachlichen Grund gleich zu behandeln. ${ }^{46}$ Infolgedessen ließe sich argumentieren, ein in einem Bundesgesetz niedergelegtes Gesetzes-benchmarking behandelt alle Länder gleich, obwohl deren Leistungsfähigkeit stark unterschiedlich und damit nicht vergleichbar ist. Gegen diese Argumentation spricht jedoch schon, dass dieses Mittel zur Optimierung der Rechtsetzung dient und damit auf einem sachlichen Grund - nämlich dem Ziel der Generierung des leistungsfähigsten Gesetzes verbunden mit der Einsparung von Verfahrenskosten - basiert.

Hinzu kommt, dass das $B V \operatorname{erf} G^{47}$ dem Gesetzgeber bei der Rüge mangelnder gesetzlicher Differenzierung einen weit reichenden Beurteilungsspielraum einräumt, weil die Legislative nicht alle tatsächlichen Verschiedenheiten rechtlich unterschiedlich behandeln muss (und im Übrigen wegen des in Art. 19 Abs. 1 Satz 1 GG niedergelegten Verbots von Einzelfallgesetzen auch nicht darf). ${ }^{48}$

45 Vgl. zur Herleitung Osterloh, L., in: Sachs, M. (Hg.): Grundgesetz: Kommentar, 4. Aufl., München, 2007, Art. 3, Rn. 74.

46 Starck C., in: Mangoldt, H.v./Klein, F./Starck, C. (Hg.): Kommentar zum Grundgesetz, Bd. 3, 5. Aufl., München, 2005, Art. 3, Rn. 230, 242 f.

47 BVerfGE 86, 81 ff., hier 87; 90, 226 ff., hier 240.

48 Vgl. dazu auch Osterloh, L., a. a. O., Art. 3, Rn. 83. 
Daher sind zur Bejahung einer Verletzung des Willkürverbots nach der Rechtsprechung trotz vielfacher Kritik im Schrifttum ${ }^{49}$ derart weit reichender Verschiedenartigkeiten notwendig, dass eine Gleichbehandlung einer am Gerechtigkeitsgedanken orientierten Betrachtung nicht stand hält. Sie dürften in Bezug auf das vorgeschlagene Gesetzes-benchmarking aber schon deshalb nicht gegeben sein, weil im Anwendungsbereich der Art. 74, 72 GG eine Gesetzgebungszuständigkeit der Bundesländer besteht, solange der Bund keinen Gebrauch macht. Denn infolgedessen geht die Kompetenzordnung für die dort niedergelegten Bereiche davon aus, dass die Länder trotz ihrer unterschiedlichen Leistungsfähigkeit den dortigen Aufgaben grundsätzlich eigenverantwortlich nachkommen können. Solange die Kennzahlen des Gesetzes-benchmarking so gewählt werden, dass sie die Länder nicht überfordern, dürfte daher eine Verletzung des allgemeinen Gleichheitssatzes ausscheiden.

\section{bb) Aufgabe der Eigenstaatlichkeit der Länder}

Aus dem in Art. 20 Abs. 1 GG niedergelegten Bundesstaatsprinzip folgt ein Verbot zur Aufgabe der aus dem Grundgesetz ableitbaren Eigenstaatlichkeit der Länder. Danach ist es den Ländern untersagt, ihre wesentlichen und unverzichtbaren Hoheitsrechte preiszugeben und so das föderale System Deutschlands zu zerrütten. ${ }^{50}$ Für den Anwendungsbereich der Art. 74, 72 GG ist in diesem Zusammenhang indes zu berücksichtigen, dass die Länder dort nur insoweit eigenverantwortlich sein können, wie der Bund keinen Gebrauch von den ihm zustehenden Kompetenzen gemacht hat. Wenn der Bund aber zugunsten einer Koordinierungsgesetzgebung samt benchmarking auf die Ausübung einer ihm zustehenden konkurrierenden Gesetzgebung verzichtet, so hat er - a majore ad minus - insoweit notwendig das Recht, Kennzahlen und etwaige Weisungsrechte einzuführen. ${ }^{51}$ Im Ergebnis macht somit auch das im Grundgesetz verankerte Prinzip von der Eigenstaatlichkeit der Länder dort halt, wo die Einflussnahme des Bundes der Verfassung und der darin enthaltenen Verteilung der Gesetzgebungskompetenzen entspricht. Dies gilt auch für die Zulässigkeit der im Gesetzes-benchmarking enthaltenen Weisungsrechte.

49 Rüfner, W.: Der allgemeine Gleichheitssatz als Differenzierungsgebot, in: Ziemske, B. u.a. (Hg.): Staatsphilosophie und Rechtspolitik: Festschrift für Martin Kriele zum 65. Geburtstag, München, 1997, 271-279, hier $276 \mathrm{f}$.

50 Grzeszick, B., in: Maunz, T./Dürig, G. (Begr.): Grundgesetz-Kommentar, Bd. 3, München, 46. EL 2006, Art. 20 Abs. 4, Rn. $160 \mathrm{f}$.

51 Machura, S., a. a. O., 425. 


\section{cc) Grundsatz bundesfreundlichen Verhaltens}

Außerdem könnte der ebenfalls aus dem Bundesstaatsprinzip ableitbare Grundsatz bundesfreundlichen Verhaltens einem benchmarking Grenzen setzen. Danach haben Bund und Länder die gegenseitige Pflicht, einander zu unterstützen und auf die Belange des anderen Rücksicht zu nehmen. ${ }^{52}$ Eine Verletzung der Bundestreue ist vor allem bei rechtsmissbräuchlichem Verhalten denkbar; ${ }^{53}$ ansonsten zielt dieser Grundsatz vornehmlich auf Abstimmung bzw. Koordination. $^{54}$

Überträgt man diese Vorgaben auf das Gesetzes-benchmarking, so bestehen zwar widerstreitende Rechtspositionen aufgrund der im Anwendungsbereich der Art. 74, 72 GG grundsätzlich gegebenen Gesetzgebungskompetenz der Länder und wegen der in diesem Mittel zur Rechtsetzungsoptimierung enthaltenen Weisungsrechte. Zu beachten ist aber, dass das Gesetzes-benchmarking zunächst auf eine eigenverantwortliche Rechtsetzung der Länder nach dem Motto trial (and error) setzt und dadurch deren Kompetenzen wie erwähnt schont, ja sogar Spielräume öffnet, die bei einem vollständigen Gebrauchmachen verschlossen wären. Hinzu kommt, dass diese Einschätzungsprärogativen dazu dienen, die best practice möglicher Landesgesetzgebung zu ermitteln. Denn sie basieren infolgedessen auf einem sachlichen Grund, so dass die Einführung eines Gesetzes-benchmarking nicht als rechtsmissbräuchliches Verhalten im oben beschriebenen Sinne eingeordnet werden kann.

Im Ergebnis steht der Grundsatz bundesfreundlichen Verhaltens einem benchmarking nicht dem Grunde nach entgegen. Er macht aber bestimmte Vorgaben für die Ausgestaltung des Gesetzes-benchmarkings. So folgt aus dem Gebot der Koordination und Zusammenarbeit, dass die Vorgabe der Kennzahlen unter Abstimmung mit den Ländern zu erfolgen hat, um deren Leistungsfähigkeit berücksichtigen zu können. Dabei bedarf es eines angemessenen, auf Ausgleich angelegten Beratungsstils, freilich ohne dass der Bund seine Interessen aufgeben müsste. Zudem verlangt das Rechtsmissbrauchsverbot, dass der Bund seine Weisungsrechte möglichst schonend einzusetzen hat und insoweit Überreaktionen

52 Roellecke, G., in: Umbach, D.C./Clemens, T. (Hg.): Grundgesetz: Mitarbeiterkommentar und Handbuch, Heidelberg, 2002, Bd. 1, Art. 20, Rn. 38 ff.

53 Bauer, H., in: Dreier, H. (Hg.): Grundgesetz: Kommentar, Bd. 2, 2. Aufl., Tübingen, 2006, Art. 20 (Bundesstaat), Rn. 42 f.; BVerfGE 1, 299 ff., hier 316.

54 Sommermann, K.-P., in: Mangoldt, H.v./Klein, F./Starck, C. (Hg.): Kommentar zum Grundgesetz, Bd. 2, 5. Aufl., München, 2005, Art. 20, Rn. 42. 
vermeiden muss. ${ }^{55}$ Außerhalb derartiger Einschränkungen ergibt sich jedoch aus dem in Art. 20 Abs. 1 GG niedergelegten Grundsatz der Bundestreue eine Pflicht der Länder, sich an Rahmenvorgaben des Bundes zu halten und darin verankerte Vorgaben umzusetzen. ${ }^{56}$

\section{dd) Wesentlichkeitsgrundsatz}

Weitere Bindungen für das Gesetzes-benchmarking ergeben sich schließlich aufgrund des aus dem Rechtsstaats- bzw. Demokratieprinzip ableitbaren Wesentlichkeitsgrundsatzes. Ihm zufolge besteht eine Pflicht zur Normierung der von den Ländern einzuhaltenden Kennzahlen in einem förmlichen Gesetz, wenn diese eine hinreichende Grundrechtssensibilität aufweisen. Dasselbe gilt für die Weisungsrechte des Bundes gegenüber den Ländern, da ihnen Zwangscharakter inne wohnt. Hinsichtlich der Voraussetzungen bietet sich dabei eine Orientierung an den Vorgaben des Art. 37 Abs. 1 GG an.

\section{ee) Schutzpflichten}

Ferner sei noch darauf hingewiesen, dass sich die Bemessung der Kennzahlen ggf. auch noch an den staatlichen Schutzpflichten zu orientieren hat. Deren Voraussetzungen und Grenzen wurden in Bezug auf den Verbraucher- und Minderjährigenschutz sowie hinsichtlich des sicherzustellenden Existenzminimums bereits oben näher erörtert, so dass an dieser Stelle auf die dortigen Ausführungen verwiesen sei.

Zudem darf in diesem Zusammenhang nicht unbeachtet bleiben, dass die staatlichen Schutzpflichten insbesondere aus Art. 2 Abs. 2 Satz 1 GG den Gesetzgeber dazu anhalten, die Auswirkungen insbesondere neuer Schutzkonzepte im Auge zu behalten.$^{57}$ Denn diese Beobachtungs- und Nachbesserungsobliegenheit verpflichtet die Legislative zur ständigen Überprüfung und Optimierung des bestehenden Rechts.

57 Calliess, C.: Die grundrechtliche Schutzpflicht im mehrpoligen Verfassungsrechtsverhältnis, in: Juristenzeitung, 61/7 (2006), 321-330; vgl. dazu auch BVerfGE 88, 203 ff., 269. 


\section{Schlussfolgerungen}

Im Ergebnis kann festgehalten werden, dass eine wie hier verstandene Koordinierungszuständigkeit des Bundes in Kombination mit einem darauf abgestimmten Länder-benchmarking ein dem Gedanken des Subsidiaritätsprinzip entsprechendes Mittel darstellt, den Ländern finanzwirksame Gestaltungs- und Innovationsspielräume zu gewähren. Dort, wo dem Bund eine konkurrierende Gesetzgebungszuständigkeit zusteht, bestünde für ihn verfassungsrechtlich bereits jetzt die Möglichkeit, sich - unter Verzicht auf eine gesetzgeberische Vollregelung im Sinne der Koordinierung auf Ziele und Leitlinien zu beschränken und im übrigen das geschilderte Verfahren eines Länder-benchmarkings einzuführen. Versteht man die Prüfung der Erforderlichkeit im Rahmen des Art. 72 Abs. 2 GG im Sinne des Verhältnismäßigkeitsprinzips, ${ }^{58}$ so wäre diese Form der Gesetzgebung ein milderes Mittel im Vergleich zur Ausschöpfung der konkurrierenden Gesetzgebungskompetenz im Sinne einer Vollregelung des Bundes.

Freilich ist in aller Regel nicht zu erwarten, dass der Bund de lege lata freiwillig auf eine ihm zustehende Möglichkeit der Vollregelung zugunsten einer bloßen Koordinierung mit Länder-benchmarking verzichtet. Daher könnte de lege ferrenda ein neuer Art. 75 GG eingeführt werden, der eine wie vorstehend definierte Koordinierungszuständigkeit des Bundes samt Länder-benchmarking als im Einzelfall zu prüfende Alternative zur konkurrierenden Gesetzgebungszuständigkeit etabliert. Als dem Leitgedanken des Innovationsföderalismus entsprechendes, im Sinne des Verhältnismäßigkeitsprinzip „milderes Mittel“ sollte dieser länderschonenden Koordinierungskompetenz ein Vorrang vor dem Gebrauchmachen konkurrierenden Zuständigkeiten eingeräumt werden. Mit anderen Worten sollte der Bund vor jedem Ausüben einer konkurrierenden Zuständigkeit auf die Prüfung verpflichtet werden, ob das angestrebte Ziel des Gesetzes nicht ebenso gut durch eine die Länder schonende Koordinierungsregelung erreicht werden kann.

\section{Standardsetzung und dezentrale Flexibilisierung/Differenzierung}

\section{Einführung}

Der Bund kann in Ausübung seiner Gesetzgebungszuständigkeiten für die Bundesländer verbindliche Standards setzen. Diese Standards beinhalten oftmals 
kostenwirksame Vorgaben des Bundes. Eine eigenständige Prioritätensetzung der Länder und Kommunen ist vor diesem Hintergrund kaum mehr möglich, ihre finanzielle Autonomie schrumpft damit zugleich auf ein Minimum. Besonders deutlich wird dies im Rahmen der Leistungsverwaltung, wenn der Bund etwa im Bereich der Kinder- und Jugendhilfe die Kategorien von Hilfeleistungen festschreibt oder in anderen Bereichen die Höhe der Leistungsansprüche exakt bestimmt. Da ein derartiges Vorgehen den Ländern die Chance gäbe, die Regulierungsdichte auf ihre spezifischen Bedürfnisse abzustimmen, dürfte der Gewinn finanzwirksamer Gestaltungs- und Innovationsspielräume außer Frage stehen.

Äußerst umstritten ${ }^{59}$ ist freilich, ob mit einer solchen Reform auch die Regionalisierung der Sozialpolitik durch entsprechende Änderungen einhergehen sollte. Auch diese könnten zu mehr Wettbewerb und Effizienz führen, sie dürften allerdings die weniger leistungsfähigen Länder keinesfalls zu einem Sozialdumping und somit zu einer Vertiefung der Kluft zwischen arm und reich nötigen. Es gälte zu verhindern, dass einzelne Länder Sozialleistungen herunterfahren, nur um nicht zum Wohlfahrtsmagneten für Arme zu werden. Dem könnte jedoch durch das Setzen bundesweiter Mindeststandards ${ }^{60}$ oder verbindlichen Entwicklungszielen (siehe oben unter III.) entgegengewirkt werden. ${ }^{61}$

Angesichts der Tatsache, dass es ein verfassungsrechtliches Konnexitätsprinzip bislang nicht gibt, andererseits aber Verschuldensgrenzen für die Länder eingeführt werden sollen, stellt sich die Frage, wie den Ländern trotzdem finanzielle Spielräume zur eigenverantwortlichen Einhaltung der Vorgaben eines Stabilitätspakts eröffnet werden können.

Konkret stellt sich zunächst die Frage, ob der Bund den Ländern de lege lata bereits jetzt eine künftige Abweichung von bundesgesetzlichen Standards gestatten kann, oder ob es dazu einer Öffnungsklausel in der Verfassung bedarf. Allerdings sind die insoweit bestehenden verfassungsrechtlichen Grenzen zu bestimmen.

59 Kritik etwa bei Leonardy, U.: Deutscher Föderalismus jenseits 2000: reformiert oder deformiert, in: Zeitschrift für Parlamentsfragen, 30/1 (1999), 135-162, hier 153, der einen Wettbewerb der Sozialversicherungssysteme als dem deutschen Verfassungsverständnis fremd ansieht.

60 Wachendorfer-Schmidt, U.: Föderalismus und Finanzverfassung, in: Männle, U. (Hg.): Föderalismus zwischen Konsens und Konkurrenz, Baden-Baden, 1998, 57-71, hier 69.

61 Leonardy, U., a. a. O., 152. 


\section{Verfassungsrechtliche Vorgaben für Abweichungsmöglichkeiten}

\section{a) Formelle Grenzen}

In formeller Hinsicht ist in diesem Zusammenhang zu beachten, dass der Bund derartige Spielräume zugunsten der Länder nur dort schaffen kann, wo ihm eine Gesetzgebungskompetenz zukommt. Es bedarf somit der Einschlägigkeit des Art. 73 GG oder der Art. 74, 72 Abs. 2 GG.

\section{aa) Gebrauchmachen des Bundes, Art. 72 Abs. 1 GG}

Im Falle einer konkurrierenden Zuständigkeit des Bundes kommt außerdem Art. 72 Abs. 1 GG Bedeutung zu. Danach haben die Bundesländer die Gesetzgebungskompetenz, solange und soweit der Bund von seiner Zuständigkeit keinen Gebrauch gemacht hat. Folglich kann der Bund den Ländern nur dann Abweichungsmöglichkeiten einräumen, wenn Art. 72 Abs. 1 GG nicht erfüllt ist.

Art. 72 Abs. 1 GG setzt eine erschöpfende Bundesregelung voraus. Aus dieser Voraussetzung ergibt sich, dass überhaupt etwas normiert wird. Unverbindliche Zielvorgaben oder Regelungsvorbehalte genügen insoweit nicht. ${ }^{62}$ Der $\mathrm{Ab}$ schlusscharakter einer Vorschrift bestimmt sich demgegenüber nach deren Normtext, der ratio legis bzw. der Historie und ist infolgedessen eine Frage des Einzelfalls. ${ }^{63}$ Etwas anderes gilt aber wiederum, wenn bestimmte Bereiche ausgenommen oder unbestimmte Rechtsbegriffe verwendet wurden, weil dann zumindest insoweit ein gewisser Spielraum verbleibt, dessen Ausfüllung mit Blick auf Art. 70 Abs. $1 \mathrm{GG}$ als Kompetenzverteilungsgrundsatz den Bundesländern obliegt. $^{64}$

Einem Bundesgesetz ist ebenfalls insoweit kein Abschlusscharakter immanent, als es den Ländern ausdrücklich einen Regelungsbereich im Sinne eines Vorbehalts überlässt. ${ }^{65}$ Soweit der Bund den Ländern die Möglichkeit zur Abweichung von seinen Vorschriften einräumt, bringt dies zum Ausdruck, dass eine erschöpfende bundesrechtliche Regelung gerade insoweit nicht vorliegen soll, als ein

62 Sannwald, R., a. a. O., Art. 72, Rn. 30; BVerfGE 34, 9 ff., hier 28.

63 Degenhart, C., a.a. O., Art. 72, Rn. 29; vgl. Korte, S.: Zur landesrechtlichen Regulierung von Sportwettenvermittlungen, in: Gewerbearchiv, 50/5 (2004), 188-191, hier 189.

64 Vgl. dazu näher Oeter, S., in: Mangoldt, H.v./Klein, F./Starck, C. (Hg.): Kommentar zum Grundgesetz, Bd. 2, 5. Aufl., München, 2005, Art. 72, Rn. 65 ff.

65 Degenhart, C., a. a. O., Art. 72, Rn. 32. 
Landesgesetzgeber eine qualitative Abweichung für zweckmäßig hält. Eine Sperrwirkung besteht somit im Ergebnis auch dann nicht, wenn den Ländern die Möglichkeit zur Abweichung von bundesrechtlichen Vorschriften geboten wird. ${ }^{66}$

Im Auge zu behalten ist, dass die Reichweite einer Delegation an den Landesgesetzgeber in Widerspruch zu den Vorgaben des Art. 72 Abs. 2 GG geraten kann. Denn wenn der Bund z.B. für einen Teilbereich des öffentlichen Wirtschaftsrechts im Sinne des Art. 74 Abs. 1 Nr. 11 GG ein einheitliches Gesetz erlässt und in diesem zugleich die Länder in umfassendem Maße zum Erlass abweichender Vorschriften ermächtigt, drängt sich die Frage auf, ob die Herstellung gleichwertiger Lebensverhältnisse wirklich „erforderlich“ ist. Vor dem Hintergrund des Leitgedankens eines „Gestaltungs- und Innovationsföderalismus“ scheiden insoweit aber nur pauschale Länderermächtigungen aus. Solange der Bundesgesetzgeber die grundlegenden Entscheidungen der von ihm geregelten Materie mit bundesweiter Geltungskraft selbst trifft, kann der vorstehend skizzierte Widerspruch vermieden werden. ${ }^{67}$

\section{bb) Art. 72 Abs. 3 GG als Vorbild für eine Abweichungsmöglichkeit}

Neben Art. 72 Abs. 1 GG gerät auch Art. 72 Abs. 3 GG in den Blick. Dieser bietet eine verfassungsrechtliche Chance für die Länder zur Modifikation bundesrechtlicher Vorschriften und könnte daher als Vorbild für andere bundesgesetzliche Abweichungsmöglichkeiten dienen.

Art. 72 Abs. 3 GG wird als Instrument zur Forcierung eines Gestaltungs- und Innovationswettbewerbs zwischen Bund und Bundesländern verstanden. Die Norm kommt vor allem den größeren Bundesländern zugute, weil insbesondere sie über die zur Entwicklung eigener Konzepte erforderlichen Ressourcen verfügen dürften. ${ }^{68}$ Die von Art. 72 Abs. 3 GG erfassten Regelungsbereiche betreffen die ehemals der Rahmengesetzgebungskompetenz des Bundes (z. B. Jagdwesen, Naturschutz und Landschaftspflege) unterliegenden Materien. Insoweit wird die „Erforderlichkeit“ einer bundesgesetzlichen Regelung i. S.d. Art. 72 Abs. 2 GG

66 So auch Uhle, A., in: Kluth, W. (Hg.): Föderalismusreformgesetz, Baden-Baden, 2007, Art. 72, Rn. 18; BVerfGE 35, $65 \mathrm{ff}$, hier 73 .

67 Grziwotz, H.: Partielles Bundesrecht und die Verteilung der Gesetzgebungsbefugnisse im Bundesstaat, in: Archiv des öffentlichen Rechts, 116/4 (1991), 588-605, hier 601.

68 Uhle, A., a. a. O., Art. 72, Rn. 47. 
stillschweigend vorausgesetzt, während landesgesetzliche Abweichungen trotzdem möglich bleiben.

Eine Abweichung von Bundesnormen durch landesrechtliche Vorschriften setzt nach Art. 72 Abs. 3 GG ein förmliches Parlamentsgesetz voraus, das im Übrigen verfassungs-, europa- und völkerrechtlichen Vorgaben entsprechen muss. Der abweichende Normbefehl darf sich nicht in der bloßen Nichtanwendung von Bundesrecht erschöpfen, sondern muss vielmehr einen eigenen sachlichen Inhalt haben. ${ }^{69}$ Eine Abweichung von Bundesrecht kommt nach Art. 72 Abs. 3 GG insoweit nicht in Betracht, als einer der abweichungsfesten Klammerzusätze vorliegt. Daraus resultieren insbesondere dort Probleme, wo sich die Ausnahmetatbestände in Generalklauseln ergehen - so z.B. in Bezug auf ,die allgemeinen Grundsätze des Naturschutzes“. Denn derartige Formulierungen ermöglichen keine trennscharfe Abgrenzung der abweichungsfesten Bundeskernkompetenzen. $^{70}$

Macht ein Landesgesetzgeber von seiner Abweichungsmöglichkeit Gebrauch, so wird das Bundesgesetz im Sinne eines Anwendungsvorrangs suspendiert. Es erfolgt also keine Derogation, sondern es gilt das jeweils spätere Gesetz im Sinne eines ,, lex-posterior derogat lege priori “ zwischen verschiedenen Normgebern, ${ }^{71}$ was Art. 72 Abs. 3 Satz 2 GG ausdrücklich klarstellt. Ein Fall des Art. 31 GG liegt entgegen teilweise vertretener Ansicht ${ }^{72}$ somit nicht vor, weil diese Vorschrift eine Kollision zwischen Bundes- und Landesrecht voraussetzt. ${ }^{73}$

\section{cc) Herausforderungen}

Kehrseite des beschriebenen Vorrangs nachfolgender Regelungen ist allerdings, dass eine Art Ping-Pong zulasten des Normadressaten droht, wenn der Bund im

69 Ebd., Art. 72, Rn. 51.

70 Klein, O./Schneider, K.: Art. 72 GG n.F. im Kompetenzgefüge der Föderalismusreform : ein Überblick zur Erfindung des ,generellen Abweichungsrechts“, zur Ersetzung der Rahmenvorschriften sowie zur Eröffnung einer neuen Antragsart im Verfassungsprozess, in: Deutsches Verwaltungsblatt, 121/24 (2006), 1549-1556, hier 1554; Papier, H.-J.: Aktuelle Fragen der bundesstaatlichen Ordnung, in: Neue juristische Wochenschrift, 60/30 (2007), 2145-2148, hier 2148.

71 Dessen Reinform setzt indes einheitliche Normgeber voraus; vgl. Joerden, J. C.: Logik im Recht, Berlin, 2005, 127.

72 In diese Richtung tendieren wohl Nierhaus, M./Rademacher, S.: Die große Staatsreform als Ausweg aus der Föderalismusfalle?, in: Landes- und Kommunalverwaltung, 16/9 (2006), 385-395, 389.

73 Klein, O./Schneider, K., a. a. O., 1552; Papier, H.-J., a. a. O., 2147. 
Anschluss an eine Abweichung eines Landes tätig wird, sich dann wieder das Land zur Modifikation herausgefordert fühlt usw.

Diese Konsequenz ist aber gerade Wesensmerkmal eines Innovations- und Gestaltungswettbewerbs um die beste Problemlösung und daher trotz der entstehenden Transaktionskosten aufgrund einer vermehrten Befassung der Normgeber im Ergebnis durchaus effizienzsteigernd. ${ }^{74}$ Dafür spricht auch, dass ein Landesgesetzgeber genauso gut mit dem Bundesgesetz zufrieden sein kann, deshalb gar nicht abweichend aktiv wird und die frei werdenden Kräfte anderweitig einsetzt. $^{75}$

Problematisch ist allerdings, dass der Anwendungsvorrang des (jeweiligen) Abweichungsgesetzes inhaltlich nur so weit reicht wie die jeweilige Regelung selbst. Denn daraus resultieren je Bundesland unterschiedliche Konsequenzen so z. B. in Form von nebeneinander anwendbarem Bundes- und Landesrecht bei Teilregelungen oder ausschließlich anwendbaren Landes- oder Bundesrecht bei einem Regelungsverzicht. Infolgedessen wird dem Normunterworfenen somit auf dem Gebiet der Abweichungsgesetzgebung künftig die Prüfung abverlangt, welches der für ihn geltende Rechtsbefehl ist, ohne dass er dies den in Betracht kommenden Normen selbst entnehmen kann, was die Gefahr von Unklarheiten und Abgrenzungsschwierigkeiten mit sich bringt. ${ }^{76}$ Denn die Frage nach dem Vorrang des jeweiligen Gesetzes ist nicht nur eine rein zeitliche, ${ }^{77}$ sondern vielmehr auch eine inhaltliche, weil die Reichweite etwa konkurrierender Vorschriften ausgelotet und auf den Einzelfall projiziert werden muss. Insoweit ist es erforderlich, dass die suspendierten Vorgängernormen im Abweichungsgesetz genannt werden, um dem Normadressaten die Orientierung zu erleichtern. Zur Rechtsklarheit trägt schließlich auch Art. 72 Abs. 3 Satz 2 GG bei, der eine sechsmonatige Karenzzeit für das Bundesrecht festlegt und den Ländern so hinreichend Zeit für eine Alternativregelung gibt, die dann in Kraft treten kann, ohne dass der Normanwender vorher mit dem Bundesrecht konfrontiert war. ${ }^{78}$

74 Mammen, L., a. a. O., 378.

75 Oeter, S.: Neustrukturierung der konkurrierenden Gesetzgebungskompetenz, Veränderung der Gesetzgebungskompetenz des Bundes, in: Starck, C. (Hg.): Föderalismusreform, München, 2007, 9-40, hier 16.

76 Klein, O./Schneider, K., a. a.O., 1553; Häde, U., a. a.O., 933; Papier, H.-J., a. a. O., 2147.

77 So aber Oeter, S.: Neustrukturierung der konkurrierenden Gesetzgebungskompetenz, a. a. O., 18.

78 Uhle, A., a. a. O., Art. 72, Rn. 52. 


\section{dd) Zwischenergebnis}

Aus Art. 72 Abs. 1, 72 Abs. 3 GG lässt sich somit ableiten, dass der Bund durchaus Regelungen treffen kann, durch die die Bundesländer von bundesrechtlichen Normen abweichen dürfen. Um die im Zusammenhang mit Art. 72 Abs. 3 GG deutlich werdenden Probleme zu umgehen, sind gesetzliche Abweichungsermächtigungen allerdings besonders bestimmt zu fassen. Zudem sollten im Falle eines Gebrauchmachens von der Abweichungskompetenz die geänderten Normen zitiert werden.

\section{b) Materielle Grenzen}

Fraglich bleibt aber, inwiefern materielle Grenzen für Abweichungsmöglichkeiten bestehen.

\section{aa) Postulat bundesweit einheitlicher Lebensbedingungen}

Mit Blick auf das Grundgesetz, insbesondere Art. 106 Abs. 3 Nr. 2 GG, wird behauptet, es gebe ein verfassungsrechtliches Postulat der Herstellung und Wahrung einheitlicher Lebensverhältnisse im gesamten Bundesgebiet. Eine solche Vorgabe stünde per se im Gegensatz zu jeder Nutzung von Gestaltungs- und Innovationsspielräumen durch die Länder.

In Art. 106 Abs. 3 Nr. 2 GG ist festgelegt, dass die Aufteilung der vom Aufkommen her einträglichsten Steuern zwischen Bund und Ländern insbesondere danach zu erfolgen hat, dass ,die Einheitlichkeit der Lebensverhältnisse im Bundesgebiet gewahrt wird“" ${ }^{79}$ Diese Vorgabe, so wird vertreten, formuliere einen allgemeinen Verfassungsgrundsatz und verbiete schon ganz generell jedwede Öffnungsregelungen, die zu unterschiedlichen Standards in den Ländern führen können.

Diese Sichtweise verkennt allerdings in systematischer Hinsicht, dass sich Art. 106 Abs. 3 Nr. 2 GG lediglich auf die Distribution bereits eingenommener Abgaben, nicht aber auf die Allokation der Produktionsfaktoren zum Erhalt von Abgaben bezieht. Insoweit ist Art. 72 Abs. 2 GG maßgeblich, der allenfalls Gleichwertigkeit (der Rechts- oder Wirtschaftsverhältnisse im gesamtstaatlichen Interesse) postuliert. Folglich sind Unterschiede zwischen den Ländern auf Allo-

79 Spannowsky, W.: Der Handlungsspielraum und die Grenzen regionaler Wirtschaftsförderung des Bundes, Berlin, 1987, 116; Gross, in: Deutsches Verwaltungsblatt, 84 (1969), 93 ff., hier 96. 
kationsebene in gewissem Maße gewollt und überdies dem Innovations- und Gestaltungswettbewerb förderlich, weil sie Anreize schaffen, zu den in Front liegenden Ländern aufzuschließen. In ähnlicher Weise bestimmt auch Art. 84 Abs. 1 S. 5 GG, dass der Bund nur ,,in Ausnahmefällen [...] wegen eines besonderen Bedürfnisses nach bundeseinheitlicher Regelung" bestimmte Vollzugsaspekte regeln darf. Auch diese Formulierung zeigt, dass nicht die Herstellung von Uniformität, sondern von Harmonie das Leitbild des Grundgesetzes in Bezug auf das Verhältnis der Bundesländer untereinander bildet. ${ }^{80} \mathrm{Im}$ Ergebnis lässt sich somit aus Art. 106 Abs. 3 Nr. 2 GG kein stichhaltiges Argument gegen eine bundesrechtlich motivierte Möglichkeit zur Abkehr von Bundesvorschriften zugunsten der Länder ableiten.

Anderes folgt auch nicht aus dem allgemeinen Gleichheitssatz des Grundgesetzes. Denn Art. 3 Abs. 1 GG würde im vorliegenden Zusammenhang nur dann auf einheitliche Lebensverhältnisse drängen und einer Abweichungsmöglichkeit entgegen stehen, wenn ein Vergleich der in den einzelnen Bundesländern erfolgten Abweichungsgesetzgebung dem Grunde nach möglich wäre. Dazu bedürfte es jedoch einer tauglichen Vergleichsgruppe. Insofern geht die weit überwiegende Literatur in Übereinstimmung mit der Rechtsprechung zu Recht davon aus, dass der allgemeine Gleichheitssatz nur gegenüber demselben Normgeber Geltung beanspruchen kann. Ein Vergleich verschiedener Landesnormen kommt daher nicht in Betracht. Andernfalls liefe man Gefahr, die aus dem in Art. 20 Abs. 1 GG niedergelegten Bundesstaatsprinzip ableitbare Eigenverantwortlichkeit der Bundesländer über Art. 3 Abs. 1 GG auszuhebeln. ${ }^{81}$

\section{bb) Grundrechtliche Grenzen}

Ferner können die Grundrechte, und zwar in ihrer Schutzpflichtendimension, einer bundesgesetzlich motivierten Abweichungsmöglichkeit Grenzen ziehen. Wenn z.B. der Bund den Ländern die Möglichkeit einräumen würde, beliebig von seinen Kontrolltatbeständen abzuweichen, so könnte die Gefahr bestehen, dass betroffene Unternehmen im Sinne eines forum shoppings ${ }^{82}$ dahin abwandern, wo die niedrigsten Kontrollstandards existieren. Daher dürfte zumindest bei denjenigen Tätigkeiten, die einen Bezug zur Gesundheit und körperlichen

80 Ähnl. Boysen, S.: Gleichheit im Bundesstaat, Tübingen, 2005, $119 \mathrm{ff}$.

81 Gubelt, M., in: Münch, I.v./Kunig, P. (Hg.): Grundgesetz-Kommentar, Bd. 1, 5. Aufl., München, 2000, Art. 3, Rn. 60; BVerfGE 21, 54 ff., hier 68.

82 Vgl. dazu näher Calliess, C./Korte, S., a. a. O., $17 \mathrm{f}$. 
Unversehrtheit der Bürger (z. B. im Bereich des Verbraucher- und Umweltschutzes) oder zu minderjährigen Verbrauchern aufweisen, der Spielraum für Abweichungsmöglichkeiten geringer sein. So könnte z. B. eine Beschränkung der Abweichungsmöglichkeiten auf bestimmte Berufsausübungsregeln verfassungsrechtlich geboten sein. Eine solche kommt in den Erprobungsklauseln der $\S \S 13$ GewO, 32 GastG deshalb auch folgerichtig zum Ausdruck. ${ }^{83}$

Soweit Abweichungsmöglichkeiten zugunsten der Bundesländer im Bereich der Leistungsverwaltung eingeführt werden sollen, ist zu berücksichtigen, dass das Grundrecht der Menschenwürde in Verbindung mit dem Sozialstaatsprinzips, dem einzelnen Bürger nach ständiger Rechtsprechung des BVerfG ein ökonomisches Existenzminimum gewährleistet. ${ }^{84}$ Konkret soll die in Art. 1 Abs. 1 Satz 2 Alt. 2 GG enthaltene Schutzpflicht des Staates jedem Bürger die Mindestvoraussetzungen für ein menschenwürdiges Dasein sichern. Dabei besteht einerseits zwar keine Beschränkung auf das zur notdürftigen Versorgung Erforderliche, andererseits ist aber auch keine Einebnung jedweder gesellschaftlicher Wohlstandsgefälle intendiert. ${ }^{85}$ Vielmehr realisiert sich das Existenzminimum an einer unteren Schwelle des gesellschaftlichen Niveaus. Diese Schwelle dürfen die Länder folglich im Rahmen einer bundesrechtlichen Abweichungsmöglichkeit nicht unterschreiten.

\section{Schlussfolgerungen}

Bereits jetzt können die Bundesländer unter Beachtung der vorstehend dargelegten Grenzen von Standards des Bundes abweichen. Dies gilt einerseits, wenn der Bundgesetzgeber eine solche Flexibilisierungs- bzw. Differenzierungsmöglichkeit im jeweiligen Gesetz explizit eröffnet. Dies Möglichkeit ist jedoch eher theoretischer Natur, insbesondere wenn die politische Konstellation auf Bundesebene nicht derjenigen auf Landesebene korrespondiert. Das Vertrauen in eine gesetzliche Öffnungsklausel ist insbesondere auch deswegen problematisch, als die Möglichkeit der Standardabweichung ein zentrales Ventil ist, mit Hilfe dessen die Länder den aus einer verfassungsrechtlichen Verschuldensgrenze flie-

83 Schönleiter, U.: Deregulierung im Gaststätten- und Gewerberecht, in: Gewerbearchiv, 51/9 (2005), 369371, hier 370 f.; Pöltl, R.: Deregulierung im Gaststättenrecht: Gesetzesänderungen zum 1. Juli 2005, in: Gewerbearchiv, 51/9 (2005), 353-364, hier 363.

84 Stober, R.: Allgemeines Wirtschaftsverwaltungsrecht, 15. Aufl., Stuttgart, 2006, § 6 II 1; BVerfGE 99, $216 \mathrm{ff}$., hier 233.

85 Kunig, P., in: Münch, I.v./Kunig, P. (Hg.): Grundgesetz-Kommentar, Bd. 1, 5. Aufl., München, 2000, Art. 1, Rn. 30, 36. 
Benden Druck regulieren können. Daher ist eine verfassungsrechtliche Regelung notwendig. Die verfassungsrechtlich gewährleistete (und damit gesicherte) Eröffnung von Abweichungsmöglichkeiten erfasst nach der Föderalismusreform I explizit aber nur die in Art. 72 Abs. 3 GG genannten Bereiche. Somit ist eine entsprechende Öffnungsklausel in den Art. 70 ff. GG vorzusehen. Diese könnte sich am Vorbild des Art. 72 Abs. 3 GG orientieren. Deren Inanspruchnahme ist an die vorstehend skizzierten verfassungsrechtlichen Grenzen gebunden; diese erschließen sich bereits in systematischer Auslegung des Grundgesetzes und müssten daher nicht zwingend nochmals ausdrücklich normiert werden. Einer solchen Öffnungsklausel steht weder der manchmal fälschlich als verfassungsrechtlicher Vorgabe gehandelte Topos der „Herstellung einheitlicher Lebensverhältnisse" noch der allgemeine Gleichheitssatz entgegen.

\section{Ergebnis}

Unter dem vorstehend entwickeltem Leitbild des Innovationsföderalismus erscheint es möglich, einer wesentlichen Voraussetzung der Föderalismusreform II zu entsprechen: Mehr Finanzverantwortung ist notwendig mit mehr Handlungsautonomie zu verbinden. Denn die Handlungsautonomie stellt gleichsam ein „Ventil“" für jenen Druck dar, der aus einer verfassungsrechtlichen Verschuldensgrenze samt Sanktionsmechanismen für die Länder (und Kommunen) notwendig folgt. Das - wie oben dargestellt - mit den föderalen Vorgaben des Grundgesetzes vereinbare Leitbild des Innovationsföderalismus - vermag eine verstärkte Handlungsautonomie der Bundesländer in Form von finanzwirksamen Gestaltungs- und Innovationsspielräumen zu legitimieren. Die in Umsetzung dieses Leitbilds vorgestellten Konzepte eines an eine Koordinierungszuständigkeit des Bundes gekoppelten Länder-Gesetzes-benchmarkings und einer bundesrechtlichen Standardsetzung mit dezentralen Flexibilisierungs- bzw. Differenzierungsmöglichkeiten zeigen beispielhaft verfassungsrechtlich zulässige Wege auf, wie den Ländern konkret mehr Handlungsautonomie gewährt werden kann. Notwendig ist damit aber auch ein Selbstverständnis der Länder, dass ihrer vom Föderalismus unterstellten Fähigkeit zur eigenverantwortlichen Aufgabenwahrnehmung, auch in finanzieller Hinsicht, korrespondiert. Treffend führt das BVerfG im Hinblick auf den horizontalen Finanzausgleich aus, es gelte „die richtige Mitte zu finden zwischen der Selbstständigkeit, Eigenverantwortlichkeit und Bewahrung der Individualität der Länder auf der einen und der solidarge- 
meinschaftlichen Mitverantwortung für die Existenz und Eigenständigkeit der Bundesgenossen auf der anderen Seite. “ 86

Der Neuordnung der Kompetenzen im Zuge der Föderalismusreform I liegt ein Verständnis von eigenständigen leistungsstarken Ländern zugrunde. Um das föderale Gleichgewicht wiederherzustellen, die zu einseitige Betonung der bundesstaatlichen Solidarität samt Postulat der ,einheitlichen Lebensverhältnisse“ zu relativieren sowie die gliedstaatlichen Eigenverantwortung stärker zu betonen, erfolgt nun eine entsprechende Reform der Finanzverfassung. ${ }^{87}$ Insoweit erscheint es nicht völlig abwegig, eine Pflicht der latent überforderten und auf Dauer finanziell nicht selbstständig ,überlebensfähigen“ Länder zur Neugliederung anzunehmen. Man könnte dies als eine dem Recht auf Solidarhilfe immanente Nebenpflicht zur Selbsthilfe bezeichnen. ${ }^{88}$ Der dem Föderalismus notwendig innewohnende Grundsatz der Zulassung von (begrenzter) Ungleichheit lässt es durchaus zu, die Landesregierung und das Landesvolk selbst die Konsequenzen ihrer vermeidbaren Untätigkeit tragen zu lassen, anstatt dass der Bund und die anderen Länder auf unbestimmte Zeit dafür zahlen. ${ }^{89}$ So könnte im Ergebnis auch eine „freiwillige“ Neugliederung der Länder gefördert bzw. angestoßen werden.

86 BVerfG, Urt. v. 11.11.1999, Az. 2 BvF 2/98, 3/98, 1/99, 2/99, Abs. 291.

87 Arndt, H.-W. u. a.: Zehn Vorschläge zur Reform des deutschen Föderalismus, in: Zeitschrift für Rechtspolitik, 33/5 (2000), 201-206, hier 202; Hinsch, A.: Neugliederung des Bundesgebiets und europäische Regionalisierung, Frankfurt/M. u. a., 2002, 134.

88 So Arndt, H.-W.: Erneuerter Föderalismus: Thesen zu einer veränderten Balance zwischen Bund und Ländern, in: Männle, U. (Hg.): Föderalismus zwischen Konsens und Konkurrenz, Baden-Baden, 1998, 31-36, hier $35 \mathrm{f}$.

89 Vgl. ebd., 36. 